\title{
Inclusion of In-Situ Velocity Measurements into the UCSD Time-Dependent Tomography to Constrain and Better-Forecast Remote-Sensing Observations
}

\author{
B.V. Jackson · P.P. Hick • M.M. Bisi • J.M. Clover • \\ A. Buffington
}

Received: 30 November 2009 / Accepted: 11 February 2010 / Published online: 5 March 2010

(C) The Author(s) 2010. This article is published with open access at Springerlink.com

\begin{abstract}
The University of California, San Diego (UCSD) three-dimensional (3-D) timedependent tomography program has been used successfully for a decade to reconstruct and forecast coronal mass ejections from interplanetary scintillation observations. More recently, we have extended this tomography technique to use remote-sensing data from the Solar Mass Ejection Imager (SMEI) on board the Coriolis spacecraft; from the Ootacamund (Ooty) radio telescope in India; and from the European Incoherent SCATter (EISCAT) radar telescopes in northern Scandinavia. Finally, we intend these analyses to be used with observations from the Murchison Widefield Array (MWA), or the LOw Frequency ARray (LOFAR) now being developed respectively in Australia and Europe. In this article we demonstrate how in-situ velocity measurements from the Advanced Composition Explorer (ACE) space-borne instrumentation can be used in addition to remote-sensing data to constrain the time-dependent tomographic solution. Supplementing the remote-sensing observations with in-situ measurements provides additional information to construct an iterated solar-wind
\end{abstract}

Remote Sensing of the Inner Heliosphere

Guest Editors: M.M. Bisi and A.R. Breen

B.V. Jackson ( $\varangle)$ · P.P. Hick · M.M. Bisi · J.M. Clover · A. Buffington

Center for Astrophysics and Space Sciences, University of California, San Diego, 9500 Gilman Drive

\#0424, La Jolla, CA 92093-0424, USA

e-mail: bvjackson@ucsd.edu

P.P. Hick

e-mail: pphick@ucsd.edu

M.M. Bisi

e-mail: mmbisi@ucsd.edu

J.M. Clover

e-mail: jclover@ucsd.edu

A. Buffington

e-mail: abuffington@ucsd.edu

P.P. Hick

San Diego Supercomputer Center, University of California, San Diego, 9500 Gilman Drive \#0505,

La Jolla, CA 92093-0505, USA 
parameter that is propagated outward from near the solar surface past the measurement location, and throughout the volume. While the largest changes within the volume are close to the radial directions that incorporate the in-situ measurements, their inclusion significantly reduces the uncertainty in extending these measurements to global 3-D reconstructions that are distant in time and space from the spacecraft. At Earth, this can provide a finely-tuned real-time measurement up to the latest time for which in-situ measurements are available, and enables more-accurate forecasting beyond this than remote-sensing observations alone allow.

Keywords Radio scintillation · Velocity fields, solar wind · Coronal mass ejections, interplanetary $\cdot$ Solar wind, disturbances

\section{Introduction}

Interplanetary scintillation (IPS) observations of meter-wavelength intensity variations from point radio sources have long been a source of heliospheric remote-sensing information. IPS measures small-scale $(\sim 150 \mathrm{~km})$ density variations along the line of sight to a radio source (e.g., Hewish, Scott, and Wills, 1964; Ananthakrishnan, Coles, and Kaufman, 1980). Typically, IPS observations include sources at specific sidereal locations over a wide range of solar elongations (angular distances from the Sun). Results from the Cambridge IPS array in the UK (Houminer, 1971), show heliospheric structures that can be classified as either corotating or detached from the Sun (Gapper et al., 1982; Hewish and Bravo, 1986; Behannon, Burlaga, and Hewish, 1991).

To optimize the use of IPS measurements and produce three-dimensional (3-D) global heliospheric representations from these observations, we have developed a Computer Assisted Tomography (CAT) program (Jackson et al., 1998, 2003; Jackson, Hick, and Buffington, 2002; Hick and Jackson, 2004; Jackson and Hick, 2005) that fits observations to a solar-wind model. We fit data from the Solar-Terrestrial Environment Laboratory (STELab; Nagoya University, Japan) (Kojima and Kakinuma, 1987), and have operated a real-time forecasting system during the nine-month period each year when the STELab IPS arrays are operational (Jackson and Hick, 2005; Jackson et al., 2009b). In this forecast, IPS results are compared with velocities and densities measured in real time from the Advanced Composition Explorer (ACE) (Stone et al., 1998) Solar Wind Electron Proton Alpha Monitor (SWEPAM) (McComas et al., 1998).

Two 3-D reconstruction techniques are used. The first technique, a corotational one, fits a heliosphere that remains unchanged over intervals of one solar rotation except for outward solar-wind flow. Here, solar rotation provides the primary change in perspective view for each observed location. The second technique is time-dependent, allowing time to vary with an interval (usually one day for STELab data) that is short compared with that of a solar rotation. This short interval causes the reconstructions to use outward solar-wind motion to provide perspective views of heliospheric structures as they pass the observer (Jackson and Hick, 2005 and references therein). The results and spatial and temporal resolutions from these techniques to-date are commensurate with, and limited by, the observational coverage and available signal-to-noise. This latter technique has recently been used to reconstruct heliospheric density and velocity from measurements by the Ootacamund (Ooty) Radio Telescope (ORT) in India (Bisi et al., 2008b, 2009b), and velocity from the European Incoherent SCATter (EISCAT) radar telescopes in northern Scandinavia (Bisi et al., 2007a, 2010). The present article concentrates specifically on this time-dependent technique, and how much it is improved by explicitly including in-situ velocity information in the 3-D reconstruction. 
Previous 3-D reconstructions of velocities and densities at Earth (e.g., Jackson, Hick, and Buffington, 2002; Jackson and Hick, 2005; Bisi et al., 2007b, 2009a), and at Mars (Jackson et al., 2007) have performed well in retrospective analyses, and in forecasts of ACE in-situ measurements of density and velocity. The forecasts, however, are often not as accurate as we believe to be possible, and the present work aims to rectify this by including available in-situ velocity information at Earth. This extension very accurately reproduces the ACE in-situ measurements at Earth, at the appropriate spatial and temporal resolutions of the 3-D reconstructions. It also shows that with this extension the global tomographic solution is not significantly affected. Remaining differences between the in-situ velocities at Earth and their remotely-sensed counterpart can provide insight into the 3-D structures of which they are a part and can also be used to more accurately extend spacecraft in-situ measurements to locations only accessed remotely.

Section 2 describes the tomographic program developed to fit IPS data, and extended to include the in-situ velocity measurements. Section 3 compares results for Carrington rotation (CR) 2061 using remote-sensing alone, and a combination of remote-sensing observations and in-situ velocity measurements. Section 4 measures the improvement in a forecast when including available in-situ velocity information. Section 5 presents a summary and conclusions.

\section{3-D Reconstructions Using In-Situ Velocity Measurements}

The present remote-sensing reconstruction technique provides 3-D solar-wind velocity and density by applying an inversion technique to the IPS data. For a more complete description of the time-dependent analysis using either IPS or Thomson-scattering data see Jackson et al. (2009b), and references therein. When a transient structure such as a heliospheric response to a coronal mass ejection (CME) crosses a large range of solar elongations, it is viewed from widely different directions. This changing perspective is exploited to reconstruct a 3-D time-dependent solar-wind model.

The present analysis from remote-sensing measurements alone incorporates the fact that line-of-sight (LOS) observations are dominated by contributions from material closest to the Sun, where more scattering occurs. However, no explicit assumptions are made about the distribution of velocity and density along these lines-of-sight. The inversion process begins with an assumed set of initial boundary ("source surface") conditions for the solar-wind model at 15 solar radii. The boundaries are set at a regular time cadence (usually at one-day intervals centered at local noon in Nagoya, Japan). In the initial stage, they are populated with an unstructured approximation of mass and velocity appropriate for that distance from the Sun. Currently, the calculation propagates mass and velocity outward from this source surface to beyond Earth using a purely kinematic model. The IPS observations are measured as close to the Sun as $11.5^{\circ}$ elongation (beyond the strong scattering regime at $327 \mathrm{MHz}$ ), and outward until the source signal strength no longer provides scintillation measurements (for strong sources this is about $90^{\circ}$ ). Each LOS is carried out to distances of $2 \mathrm{AU}$ from Earth. The largest contribution generally comes from the closest approach of the LOS to the Sun; that point is $1 \mathrm{AU}$ when viewing close to the Sun, and close to Earth for elongations $>60^{\circ}$. Typically, beyond $2 \mathrm{AU}$, remaining solar-wind contributions are less than $5 \%$ of the average total, and can be neglected. However, the calculation of the 3-D solar-wind density and velocity is continued out to at least 3 AU from the Sun. This model assumes outward radial flow and enforces conservation of mass and mass flux (Jackson et al., 1998, 2009b; Jackson and Hick, 2005). 
Figure 1 IPS $327 \mathrm{MHz}$ LOS weighting with distance from Earth as described by Jackson $e t$ al. (1998).

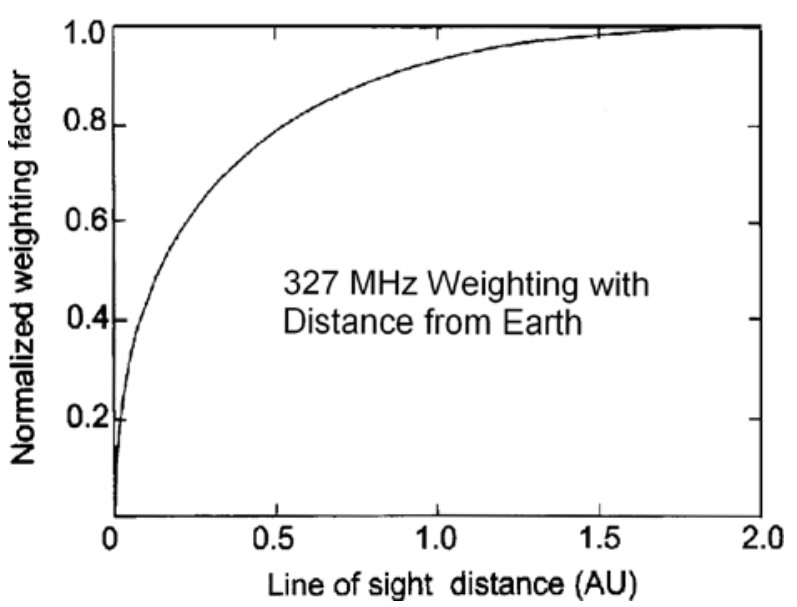

From the reconstructions, model LOS IPS scintillation levels and velocities are generated to compare with observations. Differences between observed and modeled quantities are "back-projected" by tracing each solar-wind packet within the 3-D model back to its origin on the source surface where they are used to update the boundary mass and velocity distributions. The tomographic inversion iteratively fits the model to the observations until a least-squares minimum of the differences between observations and model values is obtained. Convergence is declared when differences no longer change by more than a few percent. This usually occurs well within the maximum allowed 18 iterations.

Since only a few hundred lines-of-sight exist in any one given CR from the STELab data, a $20^{\circ} \times 20^{\circ}$ latitude and longitude with a one-day temporal digital cadence resolution is maintained by a set of Gaussian filters having 1/e constants of $14^{\circ}$ and 0.75 days respectively for the time-dependent model. This resolution is good enough to determine the large-scale transient structure of solar-wind velocity and density. Tests show that after a few iterations, any specific residue of the initial boundary conditions is erased. The technique has been used to successfully analyze CME-associated velocity and density structures using both IPS and Thomson-scattering observations (e.g., Jackson and Hick, 2005; Bisi et al., 2008a; Jackson et al., 2008), and these compare favorably with other techniques used to invert the IPS data to show the extent of CME structures (Tokumaru et al., 2005, 2007).

Two modes of time-dependent program operation are generally used at UCSD; retrospective and forecast analyses. The first maps the heliosphere using all available data covering an interesting period of observation, including data both prior to and well after the ejected solar material moves past one AU. For analysis of a single Carrington rotation we typically incorporate LOS IPS observations from a half rotation prior to the beginning of the particular Carrington rotation to a half rotation beyond its end, thus over a two-rotation time span. Forecast analysis incorporates LOS observations from a half rotation prior to the analysis period of interest, but can not utilize observations beyond a specific "forecast time". Slight differences between the retrospective and forecast analyses result from the use of different input data.

For the analyses presented in this article, we use ACE Level-0 hourly-averaged data since these are readily-available in real time for use in space-weather forecasting. Remotelysensed IPS velocity observations are formed from a weighted mean of the perpendicular solar-wind speed along the LOS (Figure 1). At Earth, the actual solar-wind velocity direction is generally far from perpendicular to the radio source LOS. In-situ velocity observa- 
tions are assumed to give a single radial outflow speed at Earth. The in-situ measurements are integrated into the reconstruction as a "line-of-sight" with a single LOS segment close to Earth. Weighting of in-situ measurements relative to the remotely-sensed data set is somewhat arbitrary. For this article we have chosen a weighting for each in-situ one-hour average measurement that is ten times the total weight of each LOS observation. Since there are approximately 375 LOS velocity observations used during the 27-day CR2061 interval (thus approximately 14 lines of sight per day), the in-situ weighting dominates that of the remotesensing value by about a factor of five. This weighting for the in-situ data measurement ensures that in-situ velocities are accommodated in the analysis as well as the reconstruction resolutions can provide. However, this high weighting leaves little ability for the leastsquares fit to depart from the in-situ velocity values which may themselves have significant error. The study of the best weighting to use for a given in-situ near-Earth monitor relative to the remote-sensing values for these 3-D reconstructions is beyond the scope of the current article.

\section{Comparison of Remote-Sensing and Remote-Sensing Plus In-Situ Inclusion}

We compare retrospective analysis of CR2061 using the time-dependent 3-D reconstruction technique described in the previous section with IPS observations, both without and with incorporating ACE Level-0 data. Figure 2 gives a velocity time series and its correlation for both cases. For comparison with the in-situ data at resolutions commensurate with the 3-D reconstructions, the ACE data have been "boxcar averaged" using a one-day-interval filter. The correlation with in-situ velocity during this particular Carrington rotation is relatively poor and often higher correlations for specific periods near CMEs and/or other whole CRs are reported (i.e., Jackson et al., 2003; Dunn et al., 2005; Bisi et al., 2009a) using STELab velocity reconstructions. On the other hand $3-\mathrm{D}$ density reconstructions from scintillation $\mathrm{g}-$ level (normalized scintillation level) have a correlation of 0.6 for this rotation, and are much better than those of velocity. As expected, there is a strikingly higher correlation (from 0.231 to 0.946) with ACE in-situ velocity measurement results for the reconstructions that include in-situ data (Figure 2c, d). Figure 3 gives a remote observer's view of the velocity 3-D reconstruction at the middle of CR2061 for both cases. Little difference is discerned between the two global velocity distributions. However, to emphasize these differences, we subtract the volume of Figure $3 \mathrm{a}$ from that of Figure $3 \mathrm{~b}$ and show this difference at a more enhanced scale in Figure 4. While the velocity volumetric difference is shown to be significant at the Earth location at the time chosen, there is little global difference in the 3-D reconstruction result elsewhere.

At this same time, the twin Solar-TErrestrial RElations Observatory (STEREO) spacecraft (Kaiser et al., 2008) are over $15^{\circ}$ from Earth, and thus at the edge of the nearest $20^{\circ}$ spatial resolution element of the near-Earth volume. Although only ACE in-situ velocity measurements are included in the present analyses during this time interval, the correlation comparisons of velocity from the Plasma and Suprathermal Ion Composition (PLASTIC) instrumentation (Galvin et al., 2008) on board STEREO increase from 0.221 to 0.698 , and from 0.080 to 0.793 , respectively, for STEREO A and STEREO B measurements.

These comparisons show, as expected, that the inclusion of in-situ measurements into the 3-D time-dependent analyses significantly improves the 3-D velocity reconstruction near the vicinity at Earth. This extends along the radial direction outward from the source surface to beyond the in-situ measurements. The lines of sight in the vicinity of the in-situ measurements can be altered significantly, but beyond this, changes in global measurements are 


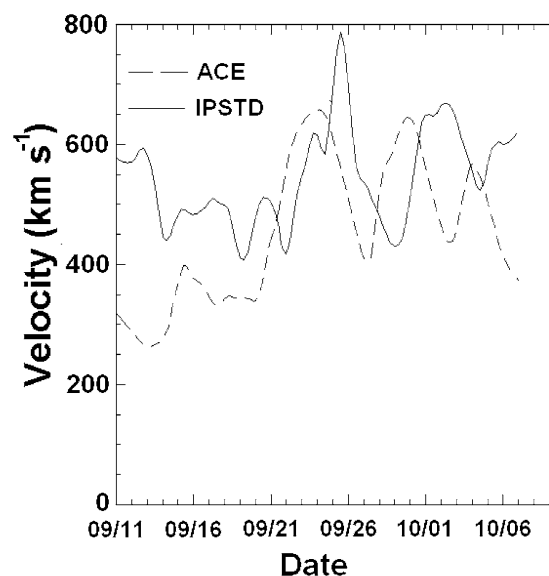

(a)

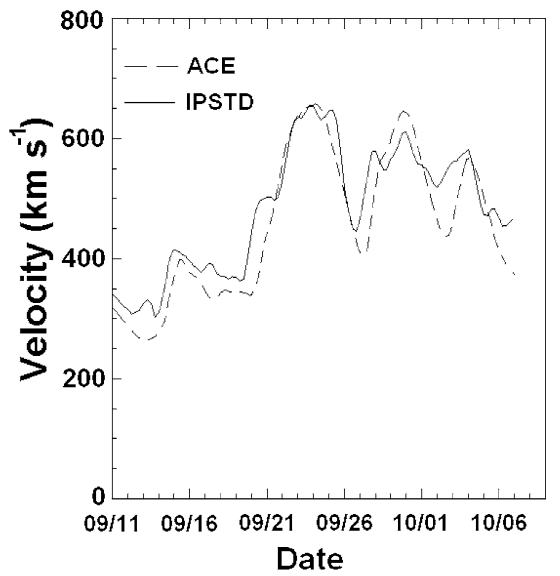

(c)

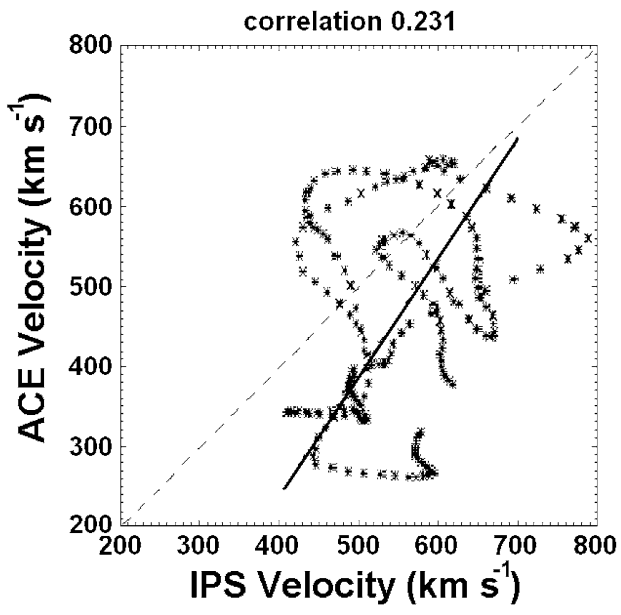

(b)

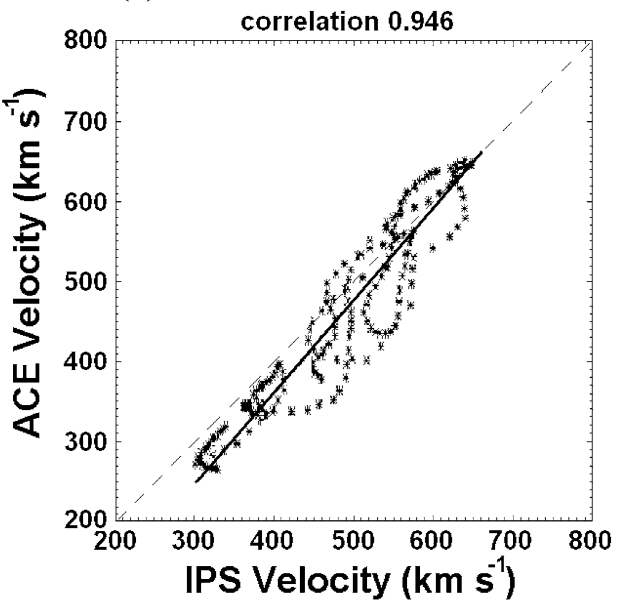

(d)

Figure 2 Velocity time series from the time-dependent 3-D reconstruction from STELab IPS data for CR2061 compared with ACE SWEPAM Level-0 data. The solid-line time series is the reconstruction result, and the dashed lines corresponds to the in-situ measurements from ACE. The ACE data have been averaged using a one-day filter to provide velocity variations with excursions comparable to the resolution available from the 3-D analyses. (a) and (b) Velocity time series and its correlation without including in-situ data in the reconstruction. (c) and (d) The same, but with the in-situ measurements incorporated.

smaller and more subtle. In the next section, we study the extent to which including in-situ measurements alters the remote-sensing analyses to produce results that are more consistent with these measurements when forecast to a future date.

\section{Velocity Forecast}

The most obvious application for incorporating in-situ measurements into the 3-D reconstructions is for its use in space-weather forecasting. If the 3-D analysis matches observed in-situ values during times when in-situ measurements exist, it is reasonable to expect that 


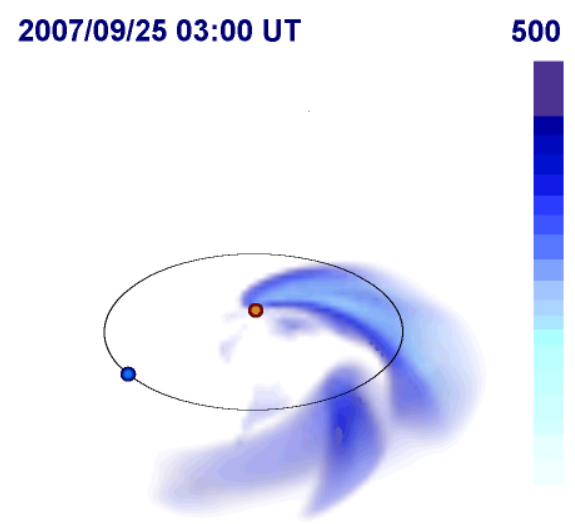

CASS ₹UCSD STEL (a)
2007/09/25 03:00 UT

500

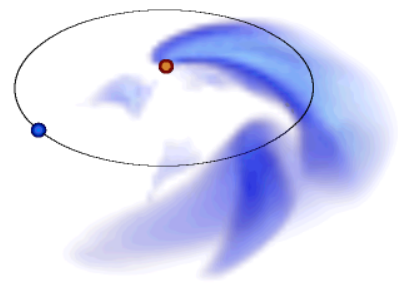

$\mathrm{V}\left(\mathrm{km} \mathrm{s}^{-1}\right)$

300

Figure 3 (a) and (b) Velocity as a remote observer would observe it from about $45^{\circ}$ west of the Sun - Earth line and $30^{\circ}$ above the ecliptic. Only the low velocities are shown, and these are located primarily in the ecliptic plane. These low velocities show some evidence of the Archimedean spiral structure often present for corotating regions in the solar wind during solar minimum. Panels (a) and (b) respectively show the result without and with including the in-situ measurements in the 3-D reconstruction.

Figure 4 Remote observer's view of the difference between 2007/09/25 03:00 UT 100 the volume presented in Figure $3 \mathrm{a}$ from the volume of Figure $3 b$. An absolute volume difference is presented with the contrast increased as shown. As noted in the text, the only significant difference in the result is near to and in the direction of the Earth from the Sun.

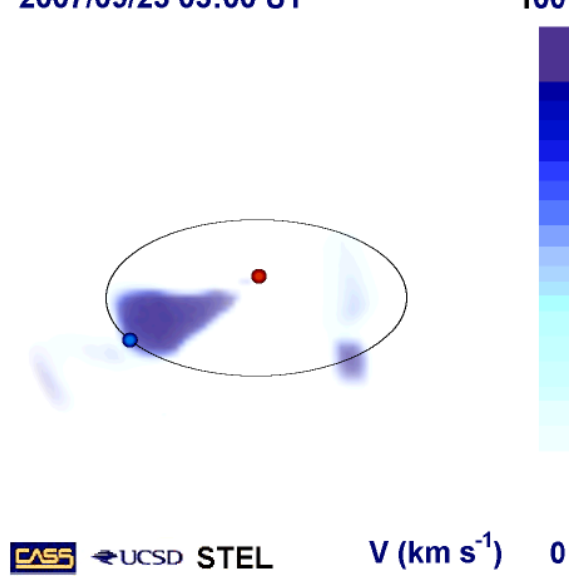

the forecast values past that time become more accurate. To demonstrate this, we operated the UCSD IPS forecast routine repeatedly, advancing the cut-off "forecast time" by one day at a time throughout CR2061 between 10 September and 6 October 2007. We chose the cut-off time each day at exactly 12:00 UT, approximately nine hours later than noon in Nagoya.

Because the radio arrays of STELab can only view their celestial meridian, and since the last radio sources observed in Japan are at best observed at approximately 18:00 h local time, each run includes IPS data up to about three hours prior to the "forecast time". The solar-wind velocity forecast 24 hours ahead of the cut-off time is determined for each run, and is compared with the actual ACE in-situ measurement (that occurs 24 hours later). 


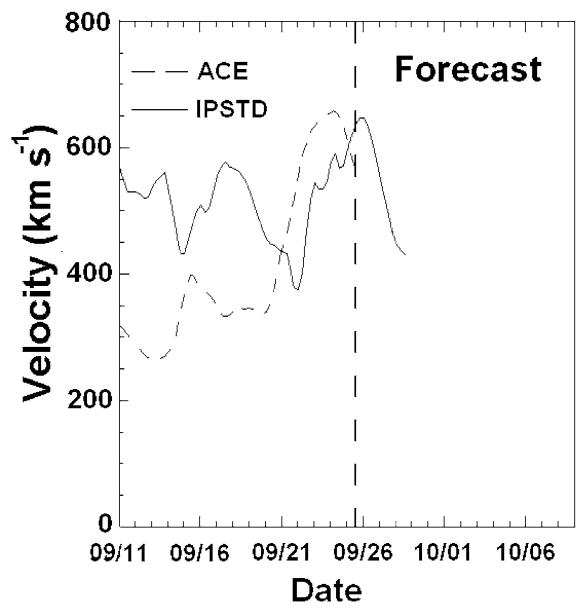

(a)

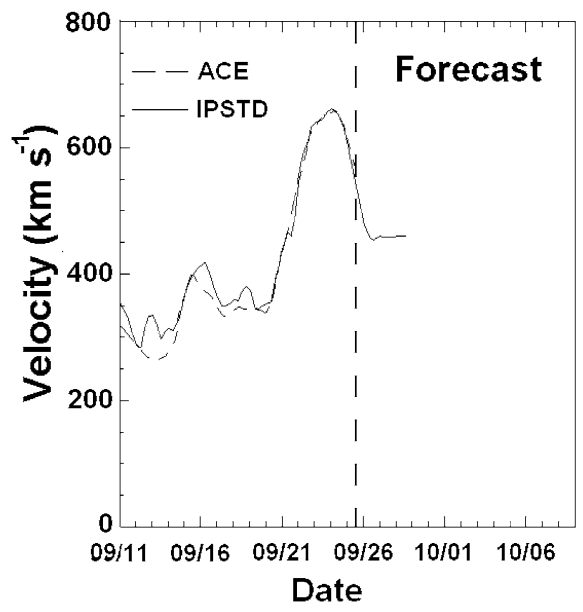

(b)

Figure 5 Velocity time series from the STELab time-dependent 3-D reconstruction forecast analysis for CR 2061 using only data prior to the cut-off time of 12:00 UT on 25 September 2007 (shown as a vertical dashed line). Forecast values appear to the right of this line. (a) Analysis where only IPS remote-sensing data are used. (b) Analysis where in-situ measurements are also included.

Figure 5 shows these results for a day at the middle of CR2061 (the same day as in Figures 3 and 4). A similar display is used in time-dependent real-time analysis shown in the current IPS space-weather forecast website at UCSD (http://ips.ucsd.edu/). As in Figures $2 \mathrm{a}$ and 2c, results from IPS reconstruction without (Figure 5a) and with (Figure 5b) in-situ data up to the cut-off time, included, are shown in comparison with ACE SWEPAM data. Past the time of forecast, only the time-dependent result is shown. As mentioned in Section 2, somewhat different (and better) results are available retrospectively when all radio source lines of sight are used. The correlations improve dramatically from a very poor 0.046 to a very high 0.991 without, and with incorporating ACE SWEPAM data in the 3-D reconstructions, respectively. Figure 6 shows the forecast values one day in advance of the cut-off time versus ACE in-situ velocity measurements for 27 days of CR2061. Deviations of the points in Figure 6 from a least-squares-fit line going through the velocity origin $(0.0,0.0)$ are measured as the square root of the variance of the IPS data values (the horizontal distance from this line). These variances are 146 and $111 \mathrm{~km} \mathrm{~s}^{-1}$ respectively for the comparisons without, and with in-situ velocities incorporated in the analysis. These variances give only one metric description of the improvement available for forecasts during CR2061. A similar variance as determined in Figure 6a has been available on the UCSD webpage for over half a decade. Forecast variance values two and three days in advance for this Carrington rotation using both comparisons with and without in-situ velocities incorporated in the analysis are summarized in Table 1 . The match between forecast velocity and in-situ measurements is consistently better if ACE data are incorporated in the tomographic analysis.

\section{Summary and Conclusions}

Combining in-situ measurements with remote-sensing observations provides a better constraint on the iterated source surface solution, which is then propagated outward through the 


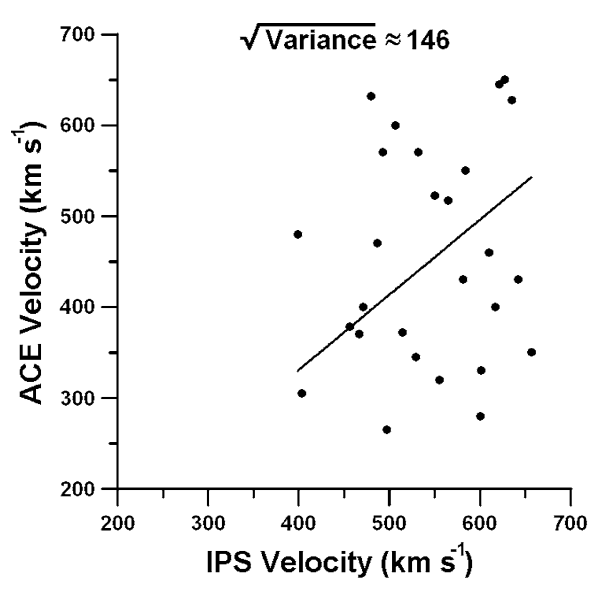

(a)

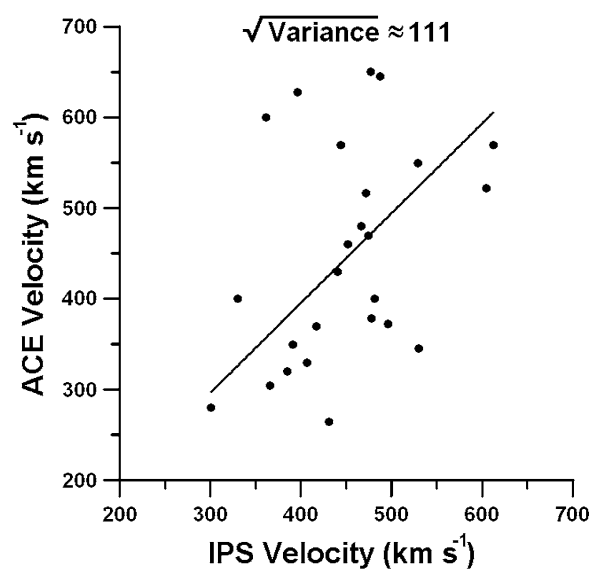

(b)

Figure 6 Correlation plots for one-day forecasts beyond the 12:00 UT cut-off time. ACE SWEPAM velocity is shown on the ordinate, and compared with the forecast value on the abscissa. A least squares line through the axis origin is drawn in the plots. (a) Analysis where no in-situ velocity is used in the 3-D time-dependent reconstructed data set. (b) Analysis where in-situ measurements have been incorporated in the 3-D reconstructions.

Table 1 Square root variance of IPS velocity deviations from the fitted line.

\begin{tabular}{llrr}
\hline & 24-hour forecast & 48-hour forecast & 72-hour forecast \\
\hline With ACE data & 111 & 106 & 86 \\
Without ACE data & 146 & 130 & 107 \\
\hline
\end{tabular}

volume using the extant solar-wind model. When doing this, the most significant changes within the volume are along the radial directions from the source surface outward past the location of the in-situ measurements, as shown for one example in Figure 4. There is also a significant increase in the correlation comparisons with STEREO PLASTIC measurements when these spacecraft are near Earth. In addition, the inclusion of in-situ measurements within the volume significantly reduces the uncertainty in extending the former measurements to global 3-D reconstructions that are distant in time and space from measurements at the spacecraft as is indicated in the forecast analyses in Figure 6 and Table 1.

It is also gratifying to note that the overall 3-D reconstructions of velocity are not greatly affected by modifying single volumetric locations over time and heavily weighting them to fit the in-situ measurements as exactly as the temporal and spatial reconstructions allow. This result attests to the robust nature of the reconstruction technique; global velocity values change little when a single measurement along a LOS is altered significantly. Of course, an essential test of the 3-D reconstruction technique requires that results incorporating the heavily-weighted in-situ data indeed fit the in-situ data; this is certified by this study. The insitu input to the 3-D reconstruction program is incorporated in the form of hourly averages, and the output of the reconstruction program is averaged spatially and temporally with the Gaussian filters mentioned in Section 2. For comparisons commensurate with the resolutions imposed by the tomographic analyses, the ACE data have been averaged using a one-day temporal filter. For this reason alone the comparisons are not expected to be exact, but only close, and this is also shown in the current study. 
For space-weather forecast analyses there is clearly a great improvement in the IPS 3-D velocity reconstruction at Earth, and in extrapolation, a better match to in-situ velocity measurements for values that are forecast. In-situ data have been the primary measurements available for study of solar-wind plasma parameters near Earth, and are thus the standard available for comparison. Even so, we do not know a priori whether the in-situ measurements are closer to the correct values than those measured remotely. The in-situ density comparison measurements near Earth of the enhancements behind shocks, using a similar 3-D reconstruction technique and Solar Mass Ejection Imager (SMEI) (Eyles et al., 2003; Jackson et al., 2004) brightness data, show large differences from in-situ monitors situated fairly close to one another (Jackson et al., 2009a). These differences in spacecraft in-situ measurements have no manifestation in the 3-D reconstruction analyses down to the resolutions available with SMEI. A determination of which spacecraft in-situ monitor gives a closer value to the large-scale remotely-sensed observations of IPS velocity is beyond the scope of this article. However, this would be an interesting study to pursue, especially when more daily observations become available from current instruments such as the new STElab Toyokawa array in Japan, the upgraded ORT, or from future instruments such as the Murchison Widefield Array (MWA) or the LOw Frequency ARray (LOFAR) now respectively under construction and initial testing in Western Australia, and in The Netherlands and other parts of Europe.

If the inclusion of in-situ measurements into the tomographic result were simply a way to smooth these so that they blend into the result determined by remote sensing alone, we would not expect much improvement in the remote-sensing forecast results beyond the immediate vicinity of the last in-situ measurements. Since this does not seem to be the case at least for this example (see Table 1), we speculate that the inclusion of the in-situ measurements near the observing point significantly improves each LOS measurement. It probably does this by refining information close to the observer, where small amounts of noise might seriously alter the result along the whole LOS.

In past analyses of densities using IPS or brightness observations, a mean in-situ level was used to provide an ambient base that was matched to the long-term trend in the observations to give the best comparisons. It is now shown possible to provide this base iteratively by incorporating the in-situ densities directly into the UCSD 3-D reconstruction technique. This has application when extending remote-sensing analysis to in-situ measurements from spacecraft such as STEREO or the MErcury Surface, Space ENvironment, GEochemistry, and Ranging (MESSENGER) mission (McNutt et al., 2006), or for that matter from measurements by future spacecraft such as the proposed NASA Solar Probe Lite or ESA's Solar Orbiter. On average, the in-situ measurements must fit those observations remotely-sensed wherever they are determined. Otherwise, the remote-sensing analyses need to be modified to accommodate these additional inputs in such matters as heliospheric structure deceleration or dissipation as dictated by the in-situ measurements. For space-weather forecast analyses it is clearly possible to use a similar approach for any plasma parameter that can be measured or inferred remotely. This includes density inferred from the IPS $g$-level proxy, or from brightness measured from electron Thomson scattering as from SMEI. It also applies to Faraday rotation observations (planned using the MWA and/or LOFAR systems) that remotely measure a combination of density and magnetic-field strength parallel to the LOS, and that have been shown to allow a 3-D inversion of the heliospheric magnetic-field vector (Jackson et al., private communication, NSF UARS-UAF Meeting presentation, September 2008, Haystack Observatory, MA, USA).

Acknowledgements B.V. Jackson, P.P. Hick, M.M. Bisi, J.M. Clover, and A. Buffington were supported at UCSD by NSF grant ATM-0331513, and more recently by NSF grant ATM-0852246 and NASA grants 
NAG5-134543 and NNG05GG45G. In addition, M.M. Bisi was supported by NSF grant ATM-0925023, and he and B.V. Jackson were both supported in part by AFOSR grant FA9550-06-1-0107. The authors wish to acknowledge and thank the group at STELab, Nagoya University (M. Kojima, M. Tokumaru, K. Fujiki, and students) for their continued support, and for making IPS data sets available under the auspices of a joint collaborative agreement between the Center for Astrophysics and Space Sciences at UCSD and STELab.

Open Access This article is distributed under the terms of the Creative Commons Attribution Noncommercial License which permits any noncommercial use, distribution, and reproduction in any medium, provided the original author(s) and source are credited.

\section{References}

Ananthakrishnan, S., Coles, W.A., Kaufman, J.J.: 1980, J. Geophys. Res. 85, 6025.

Behannon, K.W., Burlaga, L.F., Hewish, A.: 1991, J. Geophys. Res. 96, 21213.

Bisi, M.M., Jackson, B.V., Fallows, R.A., Breen, A.R., Hick, P.P., Wannberg, G., Thomasson, P., Jordan, C.A., Dorrian, G.D.: 2007a, Proc. SPIE 6689, 1.

Bisi, M.M., Jackson, B.V., Hick, P.P., Buffington, A., Clover, J.M.: 2007b, Adv. Geosci. 14, 161.

Bisi, M.M., Jackson, B.V., Hick, P.P., Buffington, A., Odstrcil, D., Clover, J.M.: 2008a, J. Geophys. Res. 113, A00A11.

Bisi, M.M., Jackson, B.V., Buffington, A., Hick, P.P., Manoharan, P.K., Clover, J.M.: 2008b, Adv. Geosci., in press.

Bisi, M.M., Jackson, B.V., Buffington, A., Clover, J.M., Hick, P.P., Tokumaru, M.: 2009a, Solar Phys. 256, 201.

Bisi, M.M., Jackson, B.V., Clover, J.M., Manoharan, P.K., Tokumaru, M., Hick, P.P., Buffington, A.: 2009b, Ann. Geophys. 27, 4479.

Bisi, M.M., Jackson, B.V., Breen, A.R., Dorrian, G.D., Fallows, R.A., Clover, J.M., Hick, P.P.: 2010, Solar Phys., this issue, submitted.

Dunn, T., Jackson, B.V., Hick, P.P., Buffington, A., Zhao, X.P.: 2005, Solar Phys. 227, 339.

Eyles, C.J., Simnett, G.M., Cooke, M.P., Jackson, B.V., Buffington, A., Hick, P.P., Waltham, N.R., King, J.M., Anderson, P.A., Holladay, P.E.: 2003, Solar Phys. 217, 319.

Galvin, A.B., Kistler, L.M., Popecki, M.A., Farrugia, C.J., Simunac, K.D.C., Ellis, L., Möbius, E., Lee, M.A., Boehm, M., Carroll, J., Crawshaw, A., Conti, M., Demaine, P., Ellis, S., Gaidos, J.A., Googins, J., Granoff, M., Gustafson, A., Heirtzler, D., King, B., Knauss, U., Levasseur, J., Longworth, S., Singer, K., Turco, S., Vachon, P., Vosbury, M., Widholm, M., Blush, L.M., Karrer, R., Bochsler, P., Daoudi, H., Etter, A., Fischer, J., Jost, J., Opitz, A., Sigrist, M., Wurz, P., Klecker, B., Ertl, M., Seidenschwang, E., Wimmer-Schweingruber, R.F., Koeten, M., Thompson, B., Steinfeld, D.: 2008, Space Sci. Rev. 136, 437.

Gapper, G.R., Hewish, A., Purvis, A., Duffett-Smith, P.J.: 1982, Nature 296, 633.

Hewish, A., Bravo, S.: 1986, Solar Phys. 106, 185.

Hewish, A., Scott, P.F., Wills, D.: 1964, Nature 203, 1214.

Hick, P.P., Jackson, B.V.: 2004, Proc. SPIE 5171, 287.

Houminer, Z.: 1971, Nat. Phys. Sci. 231, 165.

Jackson, B.V., Hick, P.P.: 2005, In: Gary, D.G., Keller, C.U. (eds.), Solar and Space Weather Radiophysics Current Status and Future Developments, ASSL 314, Springer, Dordrecht, 355.

Jackson, B.V., Hick, P.P., Buffington, A.: 2002, Proc. SPIE 4853, 23.

Jackson, B.V., Hick, P.L., Kojima, M., Yokobe, A.: 1998, J. Geophys. Res. 103, 12049.

Jackson, B.V., Hick, P.P., Buffington, A., Kojima, M., Tokumaru, M., Fujiki, K., Ohmi, T., Yamashita, M.: 2003, In: Velli, M., Bruno, R., Malara, F. (eds.) Proc. Solar Wind X, AIP Conference Proc. 679, 75.

Jackson, B.V., Buffington, A., Hick, P.P., Altrock, R.C., Figueroa, S., Holladay, P.E., Johnston, J.C., Kahler, S.W., Mozer, J.B., Price, S., Radick, R.R., Sagalyn, R., Sinclair, D., Simnett, G.M., Eyles, C.J., Cooke, M.P., Tappin, S.J., Kuchar, T., Mizuno, D., Webb, D.F., Anderson, P.A., Keil, S.L., Gold, R.E., Waltham, N.R.: 2004, Solar Phys. 225, 177.

Jackson, B.V., Boyer, J.A., Hick, P.P., Buffington, A., Bisi, M.M., Crider, D.H.: 2007, Solar Phys. 241(2), 385.

Jackson, B.V., Bisi, M.M., Hick, P.P., Buffington, A., Clover, J.M., Sun, W.: 2008, J. Geophys. Res. 113, A00A15.

Jackson, B.V., Hick, P.P., Buffington, A., Bisi, M.M., Clover, J.M., Hamilton, M.S., Tokumaru, M., Fujiki, K.: 2009a. In: Am. Inst. of Phys., Solar Wind 12 Proceedings, in press. 
Jackson, B.V., Hick, P.P., Buffington, A., Bisi, M.M., Clover, J.M., Tokumaru, M.: 2009b, Adv. Geosci., in press.

Kaiser, M.L., Kucera, T.A., Davila, J.M., St. Cyr, O.C., Guhathakurta, M., Christian, E.: 2008, Space Sci. Rev. 136, 5.

Kojima, M., Kakinuma, T.: 1987, J. Geophys. Res. 92, 7269.

McComas, D.J., Bame, S.J., Barker, P., Feldman, W.C., Phillips, J.L., Riley, P., Griffee, J.W.: 1998, Space Sci. Rev. 86, 563.

McNutt, R.L. Jr., Solomon, S.C., Gold, R.E., Leary, J.C., the MESSENGER Team: 2006, Adv. Space Res. 38, 564.

Stone, E.C., Frandsen, A.M., Mewaldt, R.A., Christian, E.R., Margolies, D., Ormes, J.F., Snow, F.: 1998, Space Sci. Rev. 86, 1.

Tokumaru, M., Kojima, M., Fujiki, K., Yamashita, M., Jackson, B.V.: 2005, In: Proceedings of the URSI GA J05-P2(0193).

Tokumaru, M., Kojima, M., Fujiki, K., Yamashita, M., Jackson, B.V.: 2007, J. Geophys. Res. 112, A05106. 